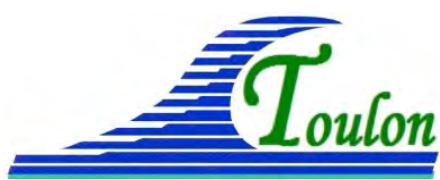

XIV èmes Journées Nationales Génie Côtier - Génie Civil

Toulon, 29 juin au $1^{\text {er }}$ juillet 2016

DOI:10.5150/jngcgc.2016.059 @ Editions Paralia CFL

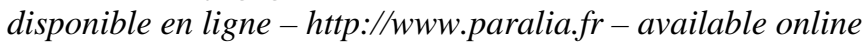

\title{
Etude hydrodynamique d'une implantation de maréliennes sur le site de la baie de Bourgneuf
}

\section{Martin SANCHEZ ${ }^{1}$, Rodica LOISEL ${ }^{2}$, Alexandre GAILLARD ${ }^{2}$, Franck SCHOEFS $^{3}$}

1. Université de Nantes, UMR-6112 CNRS - Planétologie et Géodynamique, 2 rue de la Houssinière, 44322 Nantes cedex 3, France. martin.sanchez@univ-nantes.fr

2. Université de Nantes, IEMN-IEA Institut d'Economie et de Management de Nantes, Lemna, Chemin de la Censive du Tertre, 44322 Nantes, France.

3. Université de Nantes, UMR 6183 CNRS, GeM - Institut de Recherche en Génie Civil et Mécanique, 2 rue de la Houssinière, 44322 Nantes cedex 3, France.

\section{Résumé :}

Dans l'introduction de cette note, le concept de bassins marémoteurs selon le principe des maréliennes est présenté. Par la suite, la méthode classique de Keulegan développée initialement pour examiner la propagation d'une marée sinusoïdale dans un bassin communicant avec la mer à travers un ou plusieurs chenaux, est adaptée et revisitée pour étudier (a) le marnage dans le bassin marémoteur, (b) la vitesse maximale du courant à travers les chenaux, (c) les puissances de pointe dissipée et produite, et (d) les puissances moyennes dissipée et produite. Les applications de cette méthode concernent un aménagement marémoteur en Baie de Bourgneuf selon une configuration préconisée par LEMPERIERE (2014d). Les résultats obtenus montrent que cette configuration est bien optimisée pour des conditions de marée moyenne. Cette étude montre également, que tout aménagement hydrolien à proximité d'une baie est susceptible d'entrainer une modification de l'onde de marée dont l'amortissement peut être quantifié à l'aide de la méthode développée dans cette note.

Most-clés : Maréliennes, Hydroliennes, Energies de la mer, Méthode de Keulegan, Baie de Bourgneuf.

\section{Introduction}

Annuellement dans le monde, l'hydroélectricité produit 3500 TWh alors que l'énergie de la marée qui a un potentiel équivalent ne produit dans la même période que 1 TWh (LEMPERIERE, 2014a). En fait, l'essentiel de cette énergie est produite par deux usines marémotrices : La Rance (France) et Sihwa (Corée du Sud).

Le développement récent des éoliennes avec l'implantation d'unités compétitives de 1 à $5 \mathrm{MW}$ à terre et de 2 à $10 \mathrm{MW}$ en mer, a encouragé une production d'énergie avec des hydroliennes fonctionnant sur le même principe pour exploiter les courants marins là où ils sont importants. Les seuls courants marins présentant un intérêt économique vis-àvis de cette technologie sont les courants de marée. 


\section{Thème 5 - Énergies et ressources marines}

La puissance maximale récupérable d’une hydrolienne est donnée par :

$$
P=\frac{8}{27} \rho S V^{3}
$$

où : $\rho$ est la masse volumique du fluide (environ $1025 \mathrm{~kg} / \mathrm{m}^{3}$ pour l'eau de mer), $S$ la surface du dispositif de récupération d'énergie, et $V$ la vitesse du courant. Pour une hydrolienne de $16 \mathrm{~m}$ de diamètre, la puissance maximale récupérable varie en fonction de $V$ comme suit :

- pour $V=1 \mathrm{~m} / \mathrm{s}, P=0,06 \mathrm{MW}$,

- pour $V=2 \mathrm{~m} / \mathrm{s}, P=0,49 \mathrm{MW}$,

- pour $V=3 \mathrm{~m} / \mathrm{s}, P=1,65 \mathrm{MW}$,

- pour $V=4 \mathrm{~m} / \mathrm{s}, P=3,91 \mathrm{MW}$.

Selon LAPERIERE (2014a), le potentiel mondial hydrolien utilisable à un coût acceptable, dans des sites où la vitesse de pointe des courants naturels dépasse les $3 \mathrm{~m} / \mathrm{s}$, est de l'ordre de $100 \mathrm{TWh} / \mathrm{an}$.

Une étude économique du problème montre que les hydroliennes pourraient être très rentables si elles pouvaient opérer la majeure partie du temps à une vitesse de 3,5 m/s, et ce, quel que soit le coefficient de marée. Basé sur cette constatation, LEMPERIERE (2014a) est le promoteur d'une solution innovante, désignée marélienne, consistant en la création le long du littoral de grands bassins clôturés par une digue sur laquelle de larges ouvertures alimentent des chenaux perpendiculaires dans lesquels sont placées des rangées d'hydroliennes (figure 1).

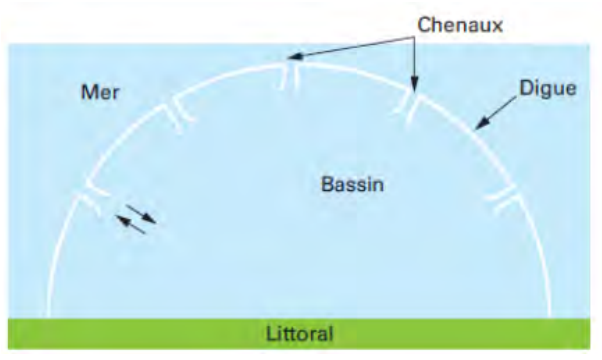

(a) Bassin clôturé par une digue

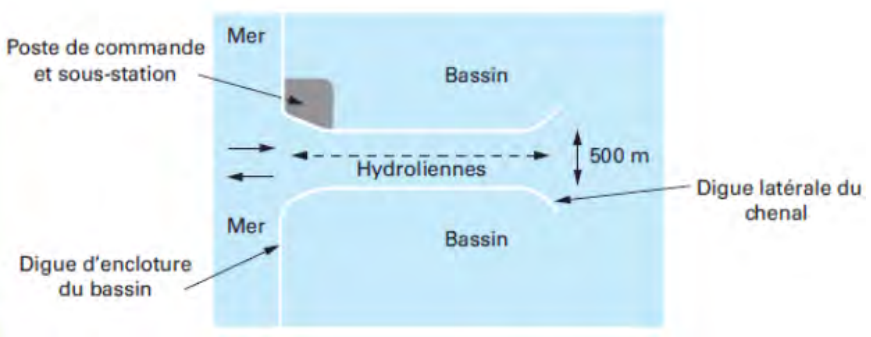

(b) Chenal d'implantation des hydroliennes.

Figure 1. Configuration d'un bassin marémoteur selon le principe des maréliennes

(LEMPERIERE, 2014a).

L'impact sur l'environnement et sur l'économie de ces aménagements possibles est abordé par LEMPERIERE (2014a ; 2014b \& 2014c). Pour la France, le potentiel sur les côtes de la Manche et de l'Atlantique est évalué à 150 TWh/an. Les deux principaux aménagements étudiés par LEMPERIERE (2014a \& 2014b) sont :

- l'aménagement de Chausey pour environ 40 TWh/an (figure 2),

- l'aménagement de la Baie de Somme pour environ 20 TWh/an (figure 3).

D’autres aménagements examinés se situent dans les secteurs Fécamp-Dieppe, Saint Vaast-Courseulles, Golfe de Saint-Brieuc et Île de Ré. 


\section{XIV èmes Journées Nationales Génie Côtier - Génie Civil \\ Toulon, 29 juin au $1^{\text {er }}$ juillet 2016}

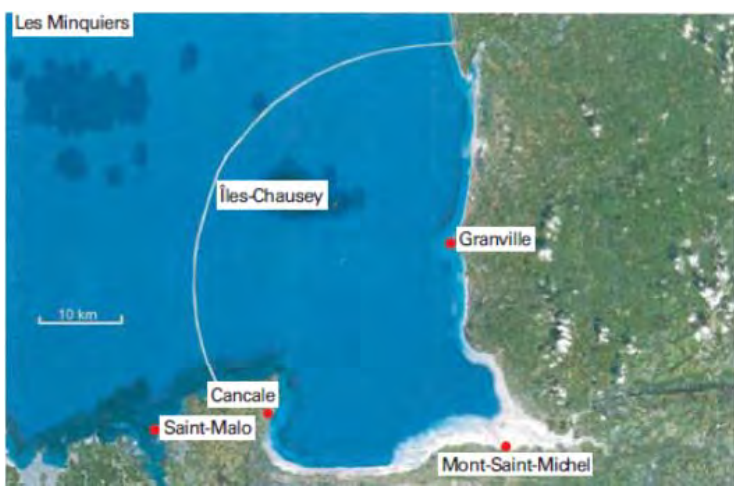

Figure 2. Aménagement de Chaussey étudié par LEMPERIERE (2014a).

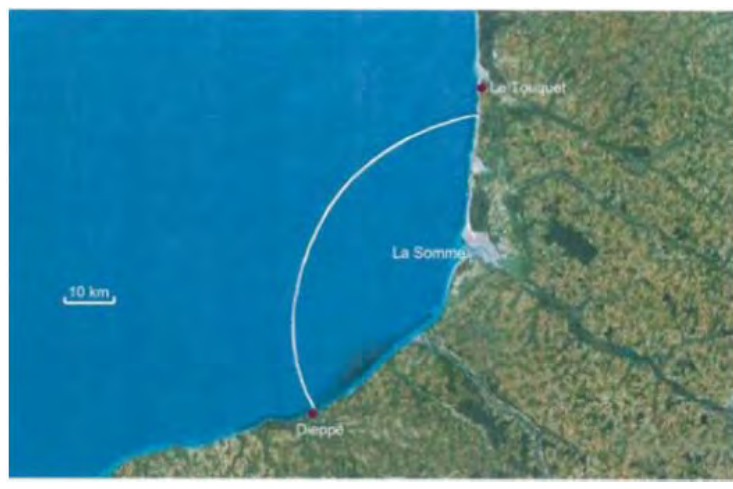

Figure 3. Aménagement de la Baie de Somme étudié par LEMPERIERE (2014a).

Les aménagements de maréliennes peuvent comporter deux variantes selon la gestion des niveaux dans le bassin :

- avec des vannes au niveau des chenaux, permettant de déconnecter le bassin de la mer afin d'engendrer des différences importantes de niveaux de la surface de l'eau entre ces deux corps d'eau,

- sans implantation de vannes laissant une libre circulation de l'eau entre le bassin et la mer.

L'objectif de cette étude est de modéliser la propagation de la marée dans un système marélien composé d'un bassin communiquant avec la mer à travers des chenaux non équipés de vannes. Cette étude doit permettre de prendre en compte les pertes d'énergie liées aux écoulements (pertes de charge hydraulique), qui ont été négligées dans les études de faisabilité de LEMPERIERE (2014a).

\section{Matériel et méthodes}

En fonction du marnage dans la partie océanique on vise à calculer :

a) le marnage dans le bassin,

b) la vitesse maximale du courant à travers les chenaux,

c) les puissances de pointe dissipée et produite,

d) les puissances moyennes dissipée et produite.

La méthode classique de KEULEGAN (1967) développée pour l'étude de systèmes lagunaires est utilisée pour aborder les points a, b et c, décrits ci-dessus.

Pour parvenir à évaluer les puissances moyennes dissipée et produite, la méthode de Keulegan est revisitée dans cette note, permettant par la même occasion de proposer 2 formulations empiriques rendant possibles les applications pratiques sans nécessité de réaliser les lectures sur les graphiques propres à cette méthode. 


\section{Thème 5 - Énergies et ressources marines}

\subsection{Théorie de Keulegan pour des systèmes bassin-mer}

Par l'application d'une équation de Bernoulli entre deux points très proches du chenal d'accès, le premier dans la partie océanique et le deuxième dans le bassin, on obtient :

$$
\left(\eta_{\text {sea }}-\eta_{\text {bay }}\right)=\left(k_{\text {Pertes }}+k_{\text {Hydrol }}\right) \frac{V|V|}{2 g}
$$

où : $\eta_{\text {sea }}$ est le niveau dans la partie océanique, $\eta_{\text {bay }}$ le niveau dans la partie abritée, $V$ la vitesse (positive pour un courant de l'océan vers le bassin), $g$ l'accélération de la pesanteur, $k_{\text {Pertes }}$ le coefficient de pertes de charge visqueuses singulières et linéiques, et $k_{\text {Hydrol }}$ un coefficient de pertes de charge traduisant l'énergie transférée vers les hydroliennes (ce dernier coefficient est introduit dans cette note pour adapter la méthode de Keulegan au problème étudié).

Si les vitesses sont élevées, le coefficient de pertes de charge singulières $k_{S}$ est lié principalement à l'effet de la mise en mouvement du fluide considéré initialement immobile (les effets des autres singularités comme l'entrée et la sortie du chenal sont négligeables et peuvent être ignorés) :

$k_{S}=1,00$

Ainsi par exemple le passage d'une vitesse nulle à une vitesse de $4 \mathrm{~m} \mathrm{~s}^{-1} \mathrm{~s}^{\text {'accompagne }}$ d'une chute de la ligne d'eau de $(1,00) \times\left(4 \mathrm{~m} \mathrm{~s}^{-1}\right)^{2} /(2 \mathrm{~g})=0,815 \mathrm{~m}$

La prise en compte des charges linéiques se fait par l'intermédiaire du coefficient $k_{L}$ suivant :

$k_{L}=\frac{2 g L}{\kappa^{2} d^{4 / 3}}$

où $\kappa$ est le coefficient de Strickler et $d$ la profondeur. On peut calculer un coefficient de pertes de charge visqueuses totales comme suit :

$k_{\text {Pertes }}=k_{S}+k_{L}$

La valeur du coefficient de pertes de charge traduisant l'énergie transférée vers les hydroliennes dépend de l'efficacité de l'ensemble des machines hydrauliques récupérant l'énergie cinétique du courant à travers les chenaux. La valeur préconisée par LEMPERIERE (2014a) est la suivante :

$k_{\text {Hydrol }}=0,667$

Le développement de Keulegan s’exprime en fonction des variables adimensionnelles suivantes :

$h_{s}=\frac{\eta_{\text {sea }}}{H_{s}} ; h_{b}=\frac{\eta_{\text {bay }}}{H_{s}} ; \theta=\frac{2 \pi t}{T}$

où $H_{s}$ représente le demi-marnage dans la partie océanique (variable selon le coefficient de marée), $t$ le temps et $T$ la période de la marée. La variation de $h_{b}$ est régie par l'équation suivante :

$\frac{d h_{b}}{d \theta}=\left\{\begin{array}{l}K\left(h_{s}-h_{b}\right)^{1 / 2}, \text { si }: h_{s} \geq h_{b} \quad(\text { pendant le flot }) \\ -K\left(h_{b}-h_{s}\right)^{1 / 2}, \text { si }: h_{b} \geq h_{s} \text { (pendant le jusant) }\end{array}\right.$ 


\section{XIV èmes Journées Nationales Génie Côtier - Génie Civil \\ Toulon, 29 juin au $1^{\text {er }}$ juillet 2016}

avec le coefficient de Keulegan $K$ défini par :

$$
K=\frac{T}{2 \pi H_{S}} \frac{a}{A}\left(\frac{2 g H_{s}}{k_{\text {Pertes }}+k_{\text {Hydrol }}}\right)^{1 / 2}
$$

Une marée sinusoïdale est modélisée par :

$h_{s}=\sin (\theta-\tau)$

où $\tau$ est la phase initiale du signal de marée (voir figure 4).

La méthode de Keulegan permet le calcul de la vitesse maximale du courant $V_{\max }$ au cours d'un cycle de marée par :

$\left|V_{\max }\right|=\frac{2 \pi H_{s}}{T} \frac{A}{a} v_{m}^{\prime}(K)$

où $v_{m}^{\prime}$ est un paramètre dont la valeur est rattachée à celle de $K$.

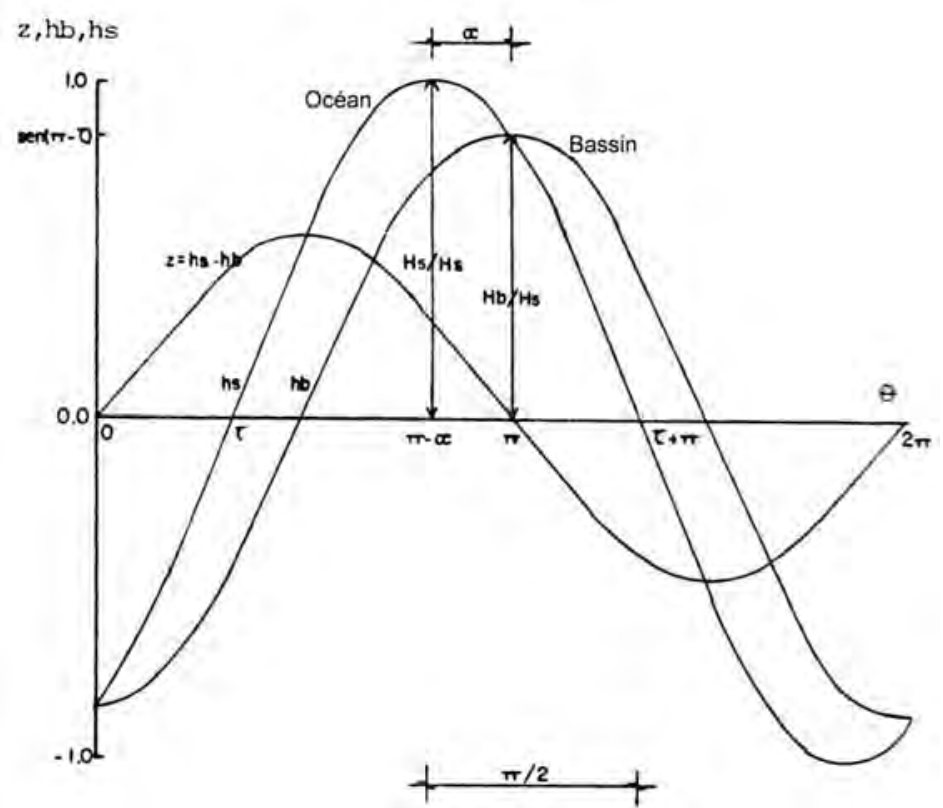

Fig. 4. Illustration de la variation des paramètres $h_{s}, h_{b}$ et $h_{s}-h_{b}$ au cours d'une marée.

2.2 Solutions de Keulegan et numérique du problème de propagation de marée

La figure 5 et 6 montrent respectivement les variations du rapport $H_{b} / H_{s}$ et du paramètre $v_{m}^{\prime}$, en fonction du coefficient de Keulegan $K$. Deux solutions de l'équation 8 y sont représentées: (i) la solution de Keulegan obtenue par un développement en série de Fourier d'ordre 3 et contenant donc des erreurs de troncature et (ii) une solution numérique obtenue dans cette étude par la méthode de Runge-Kutta à 4 étages (RK4) en divisant un cycle de marée en 3200 intervalles de temps. La solution numérique peut comporter des erreurs de calcul mais elle est vraisemblablement plus précise que la solution de Keulegan. Sur ces deux figures sont présentées respectivement les deux fonctions empiriques suivantes ajustées pour les applications pratiques : 
Thème 5 - Énergies et ressources marines

$$
\begin{aligned}
& \frac{H_{b}}{H_{s}}=\tanh (1,2 K) \\
& v_{m}^{\prime}=\tanh (K)
\end{aligned}
$$

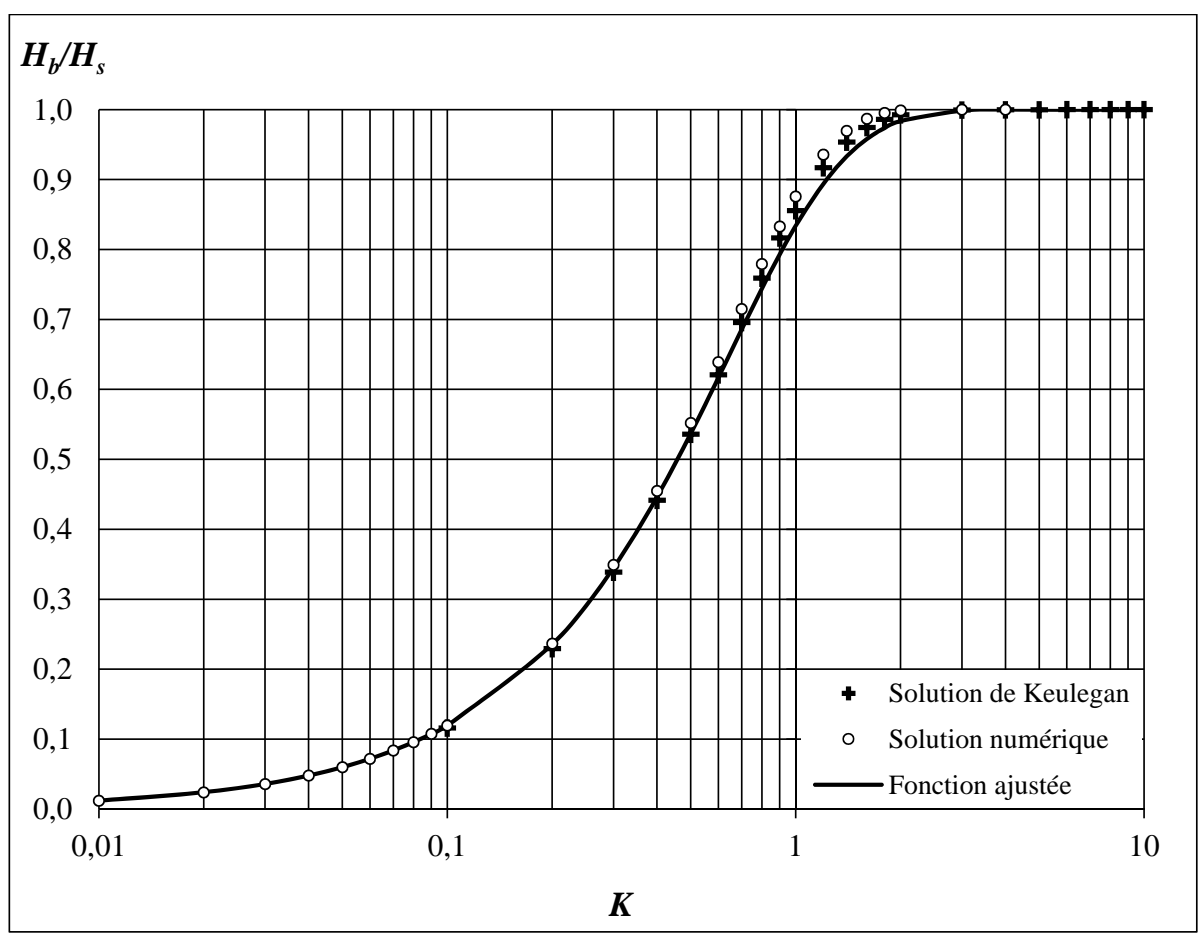

Figure 5. Variation du rapport $H_{b} / H_{s}$ en fonction du coefficient $K$ de Keulegan.

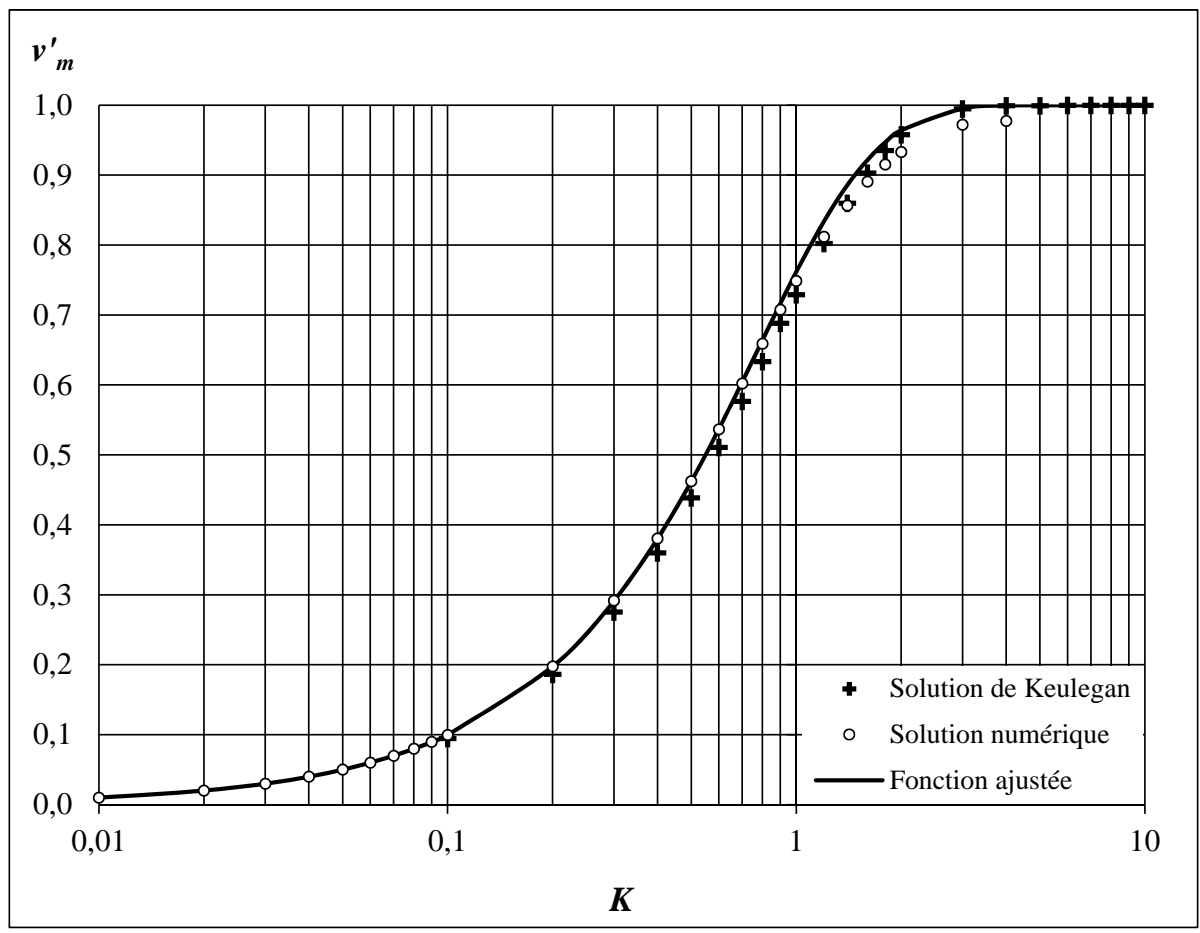

Figure 6. Variation du paramètre $v_{m}^{\prime}$ en fonction du coefficient $K$ de Keulegan. 


\section{XIV èmes Journées Nationales Génie Côtier - Génie Civil \\ Toulon, 29 juin au $1^{\text {er }}$ juillet 2016}

\subsection{Calculs des puissances}

On peut montrer que la puissance ou perte d'énergie de l'écoulement par unité de temps $\Delta t$ peut être calculée comme suit :

$\frac{\text { Energie }}{\Delta t}=\rho \times a \times\left(k_{\text {Pertes }}+k_{\text {Hydrol }}\right) \frac{V^{3}}{2}$

La puissance dissipée par viscosité est :

$\frac{\text { Energie dissipée }}{\Delta t}=\rho \times a \times\left(k_{\text {Pertes }}\right) \frac{V^{3}}{2}$

Enfin, la puissance produite ou transférée par l'écoulement vers les hydroliennes est :

$\frac{\text { Energie produite }}{\Delta t}=\rho \times a \times\left(k_{\text {Hydrol }}\right) \frac{V^{3}}{2}$

La méthode de Keulegan permet de calculer exclusivement les puissances de pointe $P_{\max }$ liées à $V_{\max }$. Le calcul des puissances moyennes $P_{\text {moy }}$, de plus d'intérêt pratique, ne sont possibles que quand les oscillations des niveaux dans le bassin sont identiques et sans décalage temporel à celles des niveaux dans la mer proche (cas pour $K>>2$ ). Dans ce cas on obtient l'expression analytique :

$\frac{P_{\text {moy }}}{P_{\max }}=\frac{4}{3 \pi}$

La figure 7 montre la variation de $P_{\text {moy }} / P_{\max }$ en fonction de $K$, résultant de la solution numérique de l'équation 8 . On constate que la solution analytique est valable pour $K>4$.

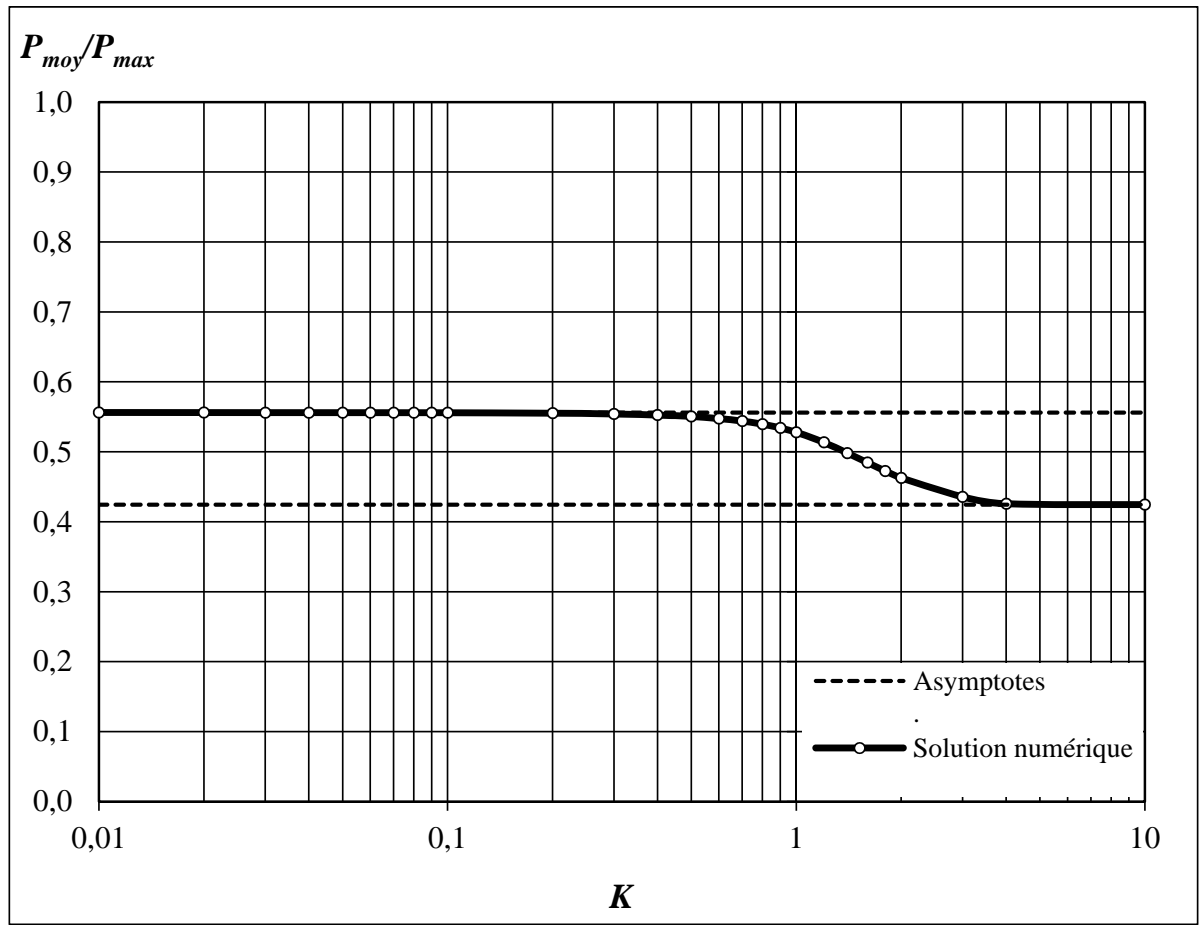

Figure 7. Variation du paramètre $v_{m}^{\prime}$ en fonction du coefficient K de Keulegan. 


\section{Application à un aménagement en Baie de Bourgneuf}

Un aménagement en Baie de Bourgneuf a été étudié par LEMPERIERE (2014d). Selon la configuration examinée, l'influence des échanges à travers la Goulet de Fromentine sur les niveaux dans la baie est négligée (on pourrait envisager de rehausser le passage du Gois d'environ 1,50 m). Une digue est implantée sur la frontière Nord-Ouest de la baie, entre le Nord de l'île de Noirmoutier et la ville Préfailles dans le continent (figure 8). Les paramètres propres à l'aménagement préconisé par LEMPERIERE (2014d) sont rassemblés dans le tableau 1 .

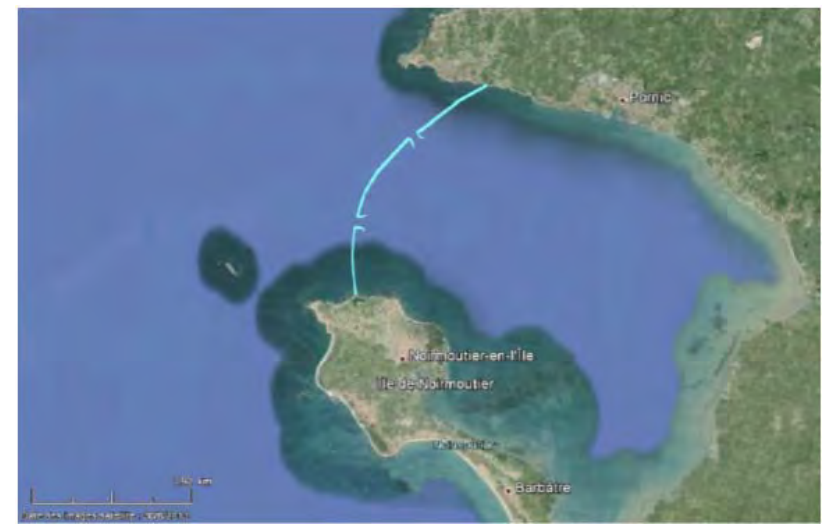

Figure 8. Illustration de l'aménagement marélien de la Baie de Bourgneuf étudié par LEMPERIERE (2014d).

Tableau 1. Paramètres caractéristiques de l'aménagement marélien de la Baie de Bourgneuf étudié par LEMPERIERE (2014d).

\begin{tabular}{llll}
\hline Notation & Définition & Valeur & Unités \\
\hline$N$ & Nombre de passes (chenaux) & 2 & \\
$B$ & Largeur de chaque chenal & 400 & $\mathrm{~m}$ \\
$D$ & Profondeur des chenaux & 18 & $\mathrm{~m}$ \\
$A$ & Section transversale totale des chenaux & 14400 & $\mathrm{~m}^{2}$ \\
$L$ & Longueur équivalente (hydraulique) de chaque chenal & 400 & $\mathrm{~m}$ \\
$A$ & Surface du bassin abrité & $250 \times 10^{6}$ & $\mathrm{~m}^{2}$ \\
\hline
\end{tabular}

\section{Résultats}

Pour les applications numériques, le coefficient de pertes de charge linéiques $k_{L}$ est négligé devant le coefficient de pertes de charge singulières $k_{S}$, si bien que l'on retient un coefficient de pertes de charge visqueuses $k_{\text {Pertes }}=k_{S}=1,00$ (voir équation 5).

Le tableau 2 montre les résultats obtenus pour 5 coefficients de marée différents. Dans ce tableau on note les données suivantes :

- La puissance moyenne théorique qui est l'énergie maximale récupérable sur un cycle de marée divisée par la période de cette dernière.

- Les résultats pour les conditions de référence sans marélienne. Quel que soit le coefficient de marée, on constate que le rapport $H_{b} / H_{s}$ est égal à 1,00 , ce qui veut dire 


\section{XIV vèmes Journées Nationales Génie Côtier - Génie Civil \\ Toulon, 29 juin au $1^{\text {er }}$ juillet 2016}

que dans les conditions naturelles l'ouverture Nord-Ouest de la Baie de Bourgneuf est suffisamment ample pour que la marée ne soit pas amortie dans cette baie. Dans tous les cas, la puissance dissipée moyenne reste négligeable devant la puissance moyenne théorique. Cette puissance dissipée provient du flux d'énergie vers la baie de Bourgneuf accompagnant la propagation naturelle de la marée.

- Les résultats pour las conditions avec marélienne selon les préconisations de LAMPERIERE (2014d) avec $k_{\text {Hydrol }}=0,667$. Le rétrécissement de l'accès à la baie s'accompagne d'un fort accroissement des vitesses maximales du courant qui atteignent $5,09 \mathrm{~m} / \mathrm{s}$ pour une marée de coefficient 115 . Les puissances dissipée et produite ne sont plus négligeables devant la puissance moyenne théorique. Cette augmentation des puissances sortant du système implique une modification du flux d'énergie naturel vers la baie de Bourgneuf. Compte tenu de la répartition statistique des coefficients de marée on peut estimer la puissance produite moyennée annuellement à environ $140 \mathrm{MW}$.

- Enfin, les résultats pour las conditions avec marélienne selon les préconisations de LAMPERIERE (2014d) mais avec une modification de la valeur suggérée pour le coefficient $k_{H y d r o l}$ dont la valeur est fixée à 1,333 (il faut noter que la valeur maximale pour $k_{\text {Hydrol }}$ n'est pas d'ordre théorique mais économique par le nombre d'éoliennes mises en place). Dans ce cas, la puissance produite moyennée annuellement vaut environ $210 \mathrm{MW}$, mais l'impact sur l'environnement devient très significatif car le marnage dans le bassin est réduit de 1/3 pour un coefficient de marée de 115.

\section{Conclusions}

Selon cette étude hydrodynamique d'une marélienne, la production d'énergie s'accompagne d'un amortissement de la marée dans le bassin qui doit être pris en compte dans les études d'impact. D’une manière plus générale, un amortissement de la marée est susceptible de se produire suite à l'implantation d'hydroliennes à l'entrée d'une baie et cet amortissement peut être quantifié par la méthode ici développée.

Cette étude montre que l'aménagement préconisé par LEMPERIERE (2014d) pour la Baie de Bourgneuf est particulièrement bien adapté pour une marée moyenne de coefficient 70 pour laquelle on obtient une vitesse maximale du courant de $3,61 \mathrm{~m} / \mathrm{s}$, un amortissement du marnage de $16 \%$ et une puissance produite moyenne de $121 \mathrm{MW}$. Au vu des résultats obtenus, il semble qu'une solution optimisée pour ce site pourrait comporter 3 chenaux de $400 \mathrm{~m}$ de largeur chacun, avec la production gérée comme suit : (i) à travers un seul chenal en marée de morte-eau, (ii) à travers deux chenaux en marée moyenne, et (iii) à travers trois chenaux en marée de vive-eau.

Selon les résultats présentés au tableau 2 du manuscrit pour $k_{H y d r o l}=0,667$, la production électrique est de 1,23 TWh/an, et l'énergie dissipée est de 1,88 TWh/an. Ces calculs correspondent à un jet d'eau à la sortie du chenal qui transporte une importante quantité d'énergie, laquelle se dissipera loin du chenal. L'optimisation du chenal marélien et de 
l'implantation des hydroliennes dans son intérieur, peut permettre de multiplier la production d'énergie par un facteur d'environ 2, par rapport aux valeurs données dans ce manuscrit. Cette optimisation implique que la vitesse de sortie du chenal doit être réduite grâce à un freinage de l'écoulement assuré par les propres hydroliennes.

Tableau 2. Tableau récapitulatif des résultats obtenus pour 5 coefficients de marée différents.

\begin{tabular}{|c|c|c|c|c|c|}
\hline Coefficient de marée & 25 & 45 & 70 & 95 & 115 \\
\hline Marnage $2 \times H_{s}(m)$ & 1,375 & 2,475 & 3,85 & 5,225 & 6,325 \\
\hline Puissance moyenne théorique (MW) & 106 & 345 & 835 & 1537 & 2253 \\
\hline \multicolumn{6}{|c|}{ Conditions de référence sans marélienne avec a=125000 $\mathrm{m}^{2}$} \\
\hline Coefficient de Keulegan $K$ & 18,977 & 14,145 & 11,341 & 9,735 & 8,848 \\
\hline$H_{b} / H_{s}$ & 1,000 & 1,000 & 1,000 & 1,000 & 1,000 \\
\hline$v_{m}^{\prime}$ & 1,000 & 1,000 & 1,000 & 1,000 & 1,000 \\
\hline$V_{\max }\left(m s^{-1}\right)$ & 0,19 & 0,35 & 0,54 & 0,74 & 0,89 \\
\hline$P_{\operatorname{moy}} / P_{\max }$ & 0,424 & 0,424 & 0,424 & 0,424 & 0,424 \\
\hline Puissance dissipée moyenne (MW) & 0,20 & 1,15 & 4,33 & 10,81 & 19,18 \\
\hline \multicolumn{6}{|c|}{ Conditions avec marélienne avec a $=14400 \mathrm{~m}^{2}$ et $k_{\mathrm{Hydrol}}=0,667$} \\
\hline Coefficient de Keulegan $K$ & 1,693 & 1,262 & 1,012 & 0,869 & 0,789 \\
\hline$H_{b} / H_{s}$ & 0,966 & 0,908 & 0,838 & 0,779 & 0,739 \\
\hline$v_{m}^{\prime}$ & 0,935 & 0,852 & 0,767 & 0,701 & 0,658 \\
\hline$V_{\max }\left(m s^{-1}\right)$ & 1,57 & 2,58 & 3,61 & 4,47 & 5,09 \\
\hline$P_{\operatorname{moy}} / P_{\max }$ & 0,479 & 0,509 & 0,527 & 0,536 & 0,540 \\
\hline Puissance dissipée moyenne (MW) & 13,68 & 64,16 & 182,33 & 354,01 & 524,24 \\
\hline Puissance produite moyenne (MW) & 9,13 & 42,79 & 121,62 & 236,13 & 349,67 \\
\hline \multicolumn{6}{|c|}{ Conditions avec marélienne avec $a=14400 m^{2}$ et $k_{H y d r o l}=1,333$} \\
\hline Coefficient de Keulegan $K$ & 1,431 & 1,067 & 0,855 & 0,734 & 0,667 \\
\hline$H_{b} / H_{s}$ & 0,938 & 0,857 & 0,772 & 0,707 & 0,664 \\
\hline$v_{m}^{\prime}$ & 0,892 & 0,788 & 0,694 & 0,626 & 0,583 \\
\hline$V_{\max }\left(m s^{-1}\right)$ & 1,50 & 2,38 & 3,26 & 3,99 & 4,51 \\
\hline$P_{\operatorname{moy}} / P_{\max }$ & 0,496 & 0,523 & 0,537 & 0,542 & 0,545 \\
\hline Puissance dissipée moyenne (MW) & 12,32 & 52,28 & 137,79 & 254,86 & 368,26 \\
\hline Puissance produite moyenne (MW) & 16,42 & 69,68 & 183,68 & 339,73 & 490,89 \\
\hline
\end{tabular}

\section{Références}

KEULEGAN G.H. (1967). Tidal flow in entrances; water-level fluctuations of basins in communication with Seas. Technical bulletin $\mathrm{n}^{\circ} 14$, Commitee on tidal hydraulics, Corps of Engineers, US Army.

LEMPERIERE F. (2014a). Utilisation innovante des hydroliennes : les maréliennes. Document de recherche RE178, Editions Techniques de l’Ingénieur, 16 p.

LEMPERIERE F. (2014b). New solution could double world tidal energy potential at half the cost. Hydropower \& Dams, Issue 1, pp 92-100.

LEMPERIERE F. (2014c). New solution could supply $200 \mathrm{GW}$ of tidal energy for 10 Asian countries. Hydropower \& Dams, Issue 2, pp 96-98.

LEMPERIERE F. (2014d). L'énergie Marémotrice pour les Pays de Loire - Le site de Bourgneuf (Noirmoutier). Rapport Hydrocoop inédit, 5 p. 